\title{
Editorial: Innovative Therapeutic and Immunomodulatory Strategies for Protozoan Infections
}

\author{
Jorge Enrique Gómez Marín ${ }^{1 *}$ and Kamal El Bissati ${ }^{2}$ \\ ${ }^{1}$ Grupo GEPAMOL, Centro Investigaciones Biomédicas, Facultad de Ciencias de la Salud, Universidad del Quindío, Armenia, \\ Colombia, ${ }^{2}$ Institute for Molecular Engineering, University of Chicago Medical Center, Chicago, IL, United States
}

Keywords: Protozoa (source: MeSH), Toxoplasma, Leishmania, Trypanosoma, Plasmodium, immunomodulation, synthetic vaccines, systems biology parasitic sensitivity tests

\section{Editorial on the Research Topic}

\section{Innovative Therapeutic and Immunomodulatory Strategies for Protozoan Infections}

In this special issue of Frontiers in Cellular and Infection Microbiology, we assembled a collection of 9 original research articles and 3 reviews within the theme "Innovative Therapeutic and Immunomodulatory Strategies for Protozoan Infections." The intended goal of this Research Topic was to present an updated view of current innovations for intervention strategies against human protozoan infections. No completely efficacious vaccines for human protozoan infections

OPEN ACCESS

Edited by: Nahed Ismail, University of Illinois at Chicago, United States

Reviewed by:

Seyed Mahmoud Hashemi, Shahid Beheshti University of Medical Sciences, Iran

*Correspondence: Jorge Enrique Gómez Marín gepamol2@uniquindio.edu.co

Specialty section:

This article was submitted to

Clinical Microbiology

a section of the journal

Frontiers in Cellular and Infection Microbiology

Received: 09 April 2019

Accepted: 30 July 2019

Published: 09 August 2019

Citation:

Gómez Marín JE and El Bissati K

(2019) Editorial: Innovative

Therapeutic and Immunomodulatory Strategies for Protozoan Infections. Front. Cell. Infect. Microbiol. 9:293.

doi: 10.3389/fcimb.2019.00293 exist. For example, the current vaccines licensed for malaria, or those currently being investigated in clinical trials, are not completely effective and their utility are controversial (Genton, 2008; Thera and Plowe, 2012). This same limitation exists with respect to availability of drugs to treat infected individuals. Often wholly effective medications are lacking, and effective medications are suboptimal. New challenge is posed by artemisinin-based combination therapy (ACT)-resistant strains of $P$. falciparum, making development of new drugs to save malaria patients ever more urgent. In ocular toxoplasmosis, complete eradication of the parasite from the host is not possible, exposing patients to persistent threat of disease reactivation (de la Torre et al., 2009). Pyrimethamine and sulfadiazine, with folinic acid, are the mainstays of treatment for toxoplasmosis, but do not treat the latent bradyzoite life-stage. In the case of South American trypanosomiasis (Chagas disease) only two drugs (benznidazole and nifurtimox) compose the armamentarium of physicians treating patients with this disease (Gomez-Marin, 2010). In light of these challenges, new research strategies have appeared that enhance the possibility of treatment and vaccine development including reverse vaccinology (Cardona et al., 2015), the bioinformatic search of second-use drug candidates (Osorio and García, 2019), and ex vivo analysis of immune response (Torres-Morales et al., 2014). This Research Topic evaluates these new possibilities and brings together authorities in this field and experiences that will feed the next wave of drugs and vaccines for this group of infections that have significant human health consequences. In toxoplasmosis, Acosta Davila et al. reviewed and summarize the utility of peripheral blood mononuclear cells (PBMCs) as a model to investigate the immunologic component of host-parasite interaction. It also delineates technical limitations associated with handling and subsequent processing of PBMCs. The authors highlight the importance of variables (e.g., time of collection of PBMC), significant implications for data interpretation and conclusions related to host-parasite interaction, and discuss some controversies related to this model and suggest possible improvements to related protocols. This ex vivo model of infection is critical to obtain accurate information about human response to infection, given notable differences in immune response in mice (Gómez-Marin, 2000). Critical to the development of new therapies against T. gondii, Rocha-Rao et al. reported the use of a thiazolodinone scaffold and its derivatives, all of which 
contain an imidazole ring, and how these compounds may lead, in the future, to improved therapies targeted against the parasite. The authors discuss in their manuscript different scaffolds that they studied and how improvements might be made, especially in terms of in silico design. Evaluation of kinase inhibitors represents a novel strategy that may overcome difficulties of cross-inhibition of host kinases. The paper of the Argentinian group, led by López et al., demonstrated interesting compounds that merit further exploration in vivo. In this work, proteins involved in DNA replication or repair are proposed as potential therapeutic targets of human pathogens. The authors provide evidence demonstrating the effect of phosphatidylinositol 3-kinase (PI3K) inhibitors (KU-55399), showing that they block Toxoplasma tachyzoite replication. They found an abolition of the phosphorylation of H2A.X, proposing that ATM kinase can be a potential drug target for abrogation of $T$. gondii growth. In the same vein, an international interdisciplinary collaborative team including the University of Chicago, the Center for Structural Genomics of Infectious Diseases at Northwestern University, and collaborators across the U.K., provides crystallographic structures of 5 Toxoplasma small enzymes function in key metabolic pathways likely to be vital for parasite survival for the international research community to use (Lykins et al.). Moreover, the group demonstrates that target-specific modulation via morpholino inhibited parasite replication in vitro. The authors hope that obtaining crystal structures of these enzymes will facilitate discovery of drugs against Toxoplasma. Looking for new compounds that block Toxoplasma at the bradyzoite stage (responsible for disease reactivation across time) remains a challenging task for scientists. Vaccine development, therefore, represents a reasonable approach to would prevent infection from becoming established. Considerable progress has been made toward identification of vaccine prototypes that are moving closer to clinical studies. This includes recombinant protein that elicits $\mathrm{CD}^{+}$and $\mathrm{CD} 4^{+} \mathrm{T}$ cells response in HLA transgenic mice (El Bissati et al., 2016); Self Assembling Protein Nanoparticles (SAPN) (El Bissati et al., 2014, 2017). RNA replicons and liposomes, amongst other methods, are other vaccine platforms reported in the literature to improve mouse survival against Toxoplasma challenges. One of the most significant challenges in vaccine development is to trigger adequate antigen presentation, requiring not only sufficient quantity of antigen, but also a stable preparation, facilitating a durable immune response. Guo et al. reported use of chitosan microspheres as a system that offers these properties and confers protection to mice against acute and chronic toxoplasmosis. The authors employed bioinformatics to screen predominant peptides that bind to $\mathrm{T}$ and $\mathrm{B}$ cells epitopes. The ones that elicited IgG were selected to compose a multigenic peptide, emulsified with chitosan and microspheres, allowing assessment for immunogenicity. Similar efforts on antigenic selection was used by Patarroyo's group from Bogota (López et al.). In this work, B- and T-cell epitopes of the $P$. vivax rhoptry neck protein 2 (PvRON2) were predicted in silico and then, the binding of predicted epitopes to HLA-DR molecules was experimentally assessed, with four peptides selected to test immunogenicity (i.e.,
T cell proliferation, cytokine production, and IgG production) in cells from Plasmodium vivax-exposed individuals from two endemic areas in Colombia. Authors concluded that two T-cell epitopes and one $\mathrm{B}$-cell epitope are promising vaccine candidates. This work illustrates the difficulties to drawing conclusions from immunological assays with human samples. In addition, Alti et al. reviewed current knowledge about the use of leptin as adjuvant. The role of leptin in immune regulation is an interesting topic that should be considered given its implications on the efficacy of vaccines in obese individuals, suggesting this factor should be included in evaluations of vaccine efficacy.

Strategies of treatment against other protozoans were also reported in this Research Topic. In malaria, given the discovery of strains of Plasmodium with intrinsic resistance to the very efficacious artemisinin (Aponte et al., 2017), new drugs are urgently needed. El Bissati et al. explored the therapeutic potential of targeting the polyamine pathway in Plasmodium and, in particular, spermidine synthase as a target of a new family of inhibitors. The polyamine pathway appears to be a valid target for further exploration and small molecule development, given its essential role in parasite survival, is likely to bear therapeutic fruit. Testing analogs effective against Microsporidia formed the basis of the authors' initial approach toward P. falciparum studies. Tetraamines and oligoamines have previously documented efficacy in experimental murine models of microsporidiosis. Surprisingly, these compounds showed no or minimal antimalarial activity. The authors found that addition of cyclohexylmethyl side groups to the tetramine backbone conferred potent inhibitory activity in both chloroquineresistant (Dd2) and chloroquine-sensitive (3D7) strains at the low nanomolar range. Furthermore, these polyamine analogs decreased levels of spermine in infected cells, suggesting a mechanism involving inhibition of spermidine synthase.

Urban transmission of trypanosomiasis and new foci for Chagas' transmission in South America have increased the interest in development of optimized treatment approaches (Rueda et al., 2014; Bello Corassa et al., 2016). The review by Osorio-Méndez and Cevallos offers perspective regarding how this might be achieved. The authors use current genetic and bioinformatics tools for identifying new molecular targets for chemotherapeutic treatment of $T$. cruzi, the causative agent of Chagas disease, and follow up with in-depth descriptions of possible targets enzymes in three metabolic pathways: synthesis of surface glycoconjugates, ergosterol biosynthesis, and neutralization of reactive oxygen species. Alberca et al. chose to focus their work on an alternative pathway, instead using a virtual ligand and structure-based screening to find polyamine analogs that could bind to the T. cruzi polyamine transporter putrescine permease TcPAT12. From the large libraries submitted for docking analysis, the authors found several hits with activities against $T$. cruzi and conclude that virtual screening is effective in finding inhibitors of putrescine uptake in T. Cruzi. In another study Gunjan et al., have studied the effect of artemisinin derivatives $(\alpha / \beta$ artemether, artesunate, and a synthetic 1, 2, 4 trioxane) on apoptotic pathway of malaria parasite and its survival. Finally, the work of the Mexican group on Giardia, Argüello-Garcia 
et al. and garlics derivatives, is an excel sum example about how nature continues offering new possibilities to find anti parasite compounds.

In conclusion, this Research Topic is a good sample of how novel chemoinformatics and bioinformatics strategies are revolutionizing the discovery and identification of new compounds. The new tools such as CRISP arrayed libraries of mutated genes have added recently new possibilities and we have seen recently an explosion of new anti-protozoa compounds. However, there is still the barrier to test such a large number of compounds, the same is occurring with the vaccines candidates

\section{REFERENCES}

Aponte, S., Guerra, Á. P., Álvarez-Larrotta, C., Bernal, S. D., Restrepo, C., González, C., et al. (2017). Baseline in vivo, ex vivo and molecular responses of Plasmodium falciparum to artemether and lumefantrine in three endemic zones for malaria in Colombia. Trans. R. Soc. Trop. Med. Hyg. 111, 71-80. doi: 10.1093/trstmh/trx021

Bello Corassa, R., Aceijas, C., Alves, P. A. B., and Garelick, H. (2016). Evolution of Chagas' disease in Brazil. Epidemiological perspective and challenges for the future: a critical review. Perspect. Public Health 137, 289-295. doi: $10.1177 / 1757913916671160$

Broutier, L., Mastrogiovanni, G., Verstegen, M. M., Francies, H. E., Gavarró, L. M., Bradshaw, C. R., et al. (2017). Human primary liver cancer-derived organoid cultures for disease modeling and drug screening. Nat. Med. 23, 1424-1435. doi: $10.1038 / \mathrm{nm} .4438$

Cardona, N. I. N. I., Moncada, D. M., and Gómez-Marin, J. E. (2015). A rational approach to select immunogenic peptides that induce IFN $-\gamma$ response against Toxoplasma gondii in human leukocytes. Immunobiology 220, 1337-1342. doi: 10.1016/j.imbio.2015.07.009

de la Torre, A., Rios, C., Cardozo, C., and Gomez-Marin, J. (2009). Frequency and factors associated with recurrences of ocular toxoplasmosis in a referral center in Colombia. Br. J. Ophthalmol. 93, 1001-1004. doi: 10.1136/bjo.2008.155861

El Bissati, K., Chentoufi, A. A., Krishack, P. A., Zhou, Y., Woods, S., Dubey, J. P., et al. (2016). Adjuvanted multi-epitope vaccines protect HLAA*11:01 transgenic mice against Toxoplasma gondii. JCI Insight 1:e85955. doi: 10.1172 /jci.insight. 85955

El Bissati, K., Zhou, Y., Dasgupta, D., Cobb, D., Dubey, J. P., Burkhard, P., et al. (2014). Effectiveness of a novel immunogenic nanoparticle platform for Toxoplasma peptide vaccine in HLA transgenic mice. Vaccine 32, 3243-3248. doi: 10.1016/j.vaccine.2014.03.092

El Bissati, K., Zhou, Y., Paulillo, S. M., Raman, S. K., Karch, C. P., Roberts, C. W., et al. (2017). Protein nanovaccine confers robust immunity against Toxoplasma. NPJ Vaccines 2:24. doi: 10.1038/s41541-017-0024-6 and it is necessary to develop efficacious selective screening methods for new drugs and vaccines. At this respect, the organoids and tissue explant-based models of infection can offer a new strategy to be developed for the evaluation of antiprotozoan drug candidates (Broutier et al., 2017). This could represent a pathway of hope to obtain better drugs and vaccines for these important human diseases.

\section{AUTHOR CONTRIBUTIONS}

JG and KE drafted and edited the editorial.

Genton, B. (2008). Malaria vaccines: a toy for travelers or a tool for eradication? Expert Rev. Vaccines 7, 597-611. doi: 10.1586/14760584.7. 5.597

Gómez-Marin, J. (2000). No NO production during human Toxoplasma infection. Parasitol. Today 16, 131. doi: 10.1016/S0169-4758(99) 01614-2

Gomez-Marin, J. E. (2010). Protozoologia Medica. First. Bogota: Manual Moderno.

Osorio, J. C. C., and García, A. M. G. (2019). Antiparasitic phytotherapy perspectives, scope and current development. Infection 23, 189-204. doi: 10.22354/IN.V23I2.777

Rueda, K., Trujillo, J. E., Carranza, J. C., and Vallejo, G. A. (2014). Transmisión oral de Trypanosoma cruzi: un nuevo escenario epidemiológico de la enfermedad de Chagas en Colombia y otros países suramericanos. Biomédica 34, 631-641. doi: 10.7705/biomedica.v34i4.2204

Thera, M. A., and Plowe, C. V. (2012). Vaccines for malaria: how close are we? Annu. Rev. Med 63, 345-357. doi: 10.1146/annurev-med-022411192402

Torres-Morales, E., Taborda, L., Cardona, N., De-la-Torre, A., Sepulveda-Arias, J. C., Patarroyo, M. A., et al. (2014). Th1 and Th2 immune response to P30 and ROP18 peptides in human toxoplasmosis. Med. Microbiol. Immunol. 203, 315-322. doi: 10.1007/s00430-014-0339-0

Conflict of Interest Statement: The authors declare that the research was conducted in the absence of any commercial or financial relationships that could be construed as a potential conflict of interest.

Copyright (c) 2019 Gómez Marín and El Bissati. This is an open-access article distributed under the terms of the Creative Commons Attribution License (CC BY). The use, distribution or reproduction in other forums is permitted, provided the original author(s) and the copyright owner(s) are credited and that the original publication in this journal is cited, in accordance with accepted academic practice. No use, distribution or reproduction is permitted which does not comply with these terms. 